\title{
Noncorticosteroid Combination Shampoo versus $1 \%$ Ketoconazole Shampoo for the Management of Mild-to-Moderate Seborrheic Dermatitis of the Scalp: Results from a Randomized, Investigator-Single-Blind Trial Using Clinical and Trichoscopic Evaluation
}

\author{
Federica Dall'Oglio Francesco Lacarrubba Anna Elisa Verzì Giuseppe Micali \\ Dermatology Clinic, University of Catania, Catania, Italy
}

\section{Key Words}

Seborrheic dermatitis · Scalp · Antifungal shampoo · Antiinflammatory shampoo $\cdot$ Ketoconazole $\cdot$ Topical treatment. Trichoscopy

\begin{abstract}
Purpose: The aim of this study was to assess the efficacy and tolerability of a combination noncorticosteroid, antiinflammatory/antifungal shampoo versus $1 \%$ ketoconazole shampoo in the treatment of mild-to-moderate scalp seborrheic dermatitis (SD). Procedures: Twenty patients were randomized to using the combination shampoo (group A, 10 patients) or the $1 \%$ ketoconazole shampoo (group B, 10 patients) 3 times a week every other day for 8 weeks. Efficacy was evaluated by measuring the degree of scaling and pruritus by clinical and trichoscopic examination using a 4-point scale. Additionally, a physician global assessment (PGA) was assessed at the end of the study. Results: At 4 weeks, there was a significant reduction of scaling from baseline for both groups, while pruritus showed a significant reduction only for group A. After 8 weeks, there was a significant reduction of scaling and pruritus for both groups. PGA showed a com-
\end{abstract}

plete response in $90 \%$ of the cases in both groups. Conclusions: The results of our study demonstrate that the combination noncorticosteroid, antiinflammatory/antifungal shampoo represents an alternative approach to standard topical treatment for scalp SD. A noncorticosteroid shampoo may be equally safe and effective as ketoconazole shampoo for scalp SD, and trichoscopy provides accurate and reliable quantifiable data to assist in therapeutic monitoring.

(c) 2015 S. Karger AG, Basel

\section{Introduction}

Seborrheic dermatitis (SD) is a common chronic, recurrent, inflammatory skin condition that most commonly affects the scalp and face of adolescent and adult patients [1-7]. Scalp SD may range from loosely adherent, small white flakes (dandruff) to yellowish, oily scales. They may accumulate in localized patches or may be distributed diffusely on the scalp surface. Erythema and pruritus are generally present $[8,9]$.

The pathogenesis of SD is still unclear, but it seems to be multifactorial, involving sebaceous gland function,

\section{KARGER 125}

(c) 2015 S. Karger AG, Base

2296-9195/15/0013-0126\$39.50/0

E-Mail karger@karger.com

www.karger.com/sad
Giuseppe Micali, MD

Dermatology Clinic, University of Catania

A.O.U. Policlinico Vittorio Emanuele

Via Santa Sofia, 78, IT-95123 Catania (Italy)

E-Mail cldermct@gmail.com 
the presence on the skin of yeasts belonging to Malassezia spp. (formerly called Pityrosporum ovale), and the individual immunological response [5-6, 10]. In particular, Malassezia yeasts act by digesting sebaceous triglycerides and producing free fatty acids which in turn promote inflammation in susceptible individuals $[2,8]$. Additional precipitating factors include neurological and degenerative disorders (Parkinson's disease), immune suppression (HIV and non-HIV related), genetic disorders (trisomy 21), as well as physical and psychological stress $[1,11]$.

Available treatments for scalp SD are intended to eradicate Malassezia spp. and to reduce inflammation and scaling $[9,12,13]$. Topical therapies are the mainstay of treatment as the condition is recurrent and responds well to these agents [2].

According to two recent Cochrane reviews, some topical drugs, including corticosteroids $(0.1-1 \%$ hydrocortisone, $0.05-0.1 \%$ betamethasone, $0.05 \%$ clobetasol, $0.1 \%$ amcinonide, $0.1 \%$ mometasone, $0.01 \%$ fluocinolone acetonide) and antifungals (1-2\% ketoconazole, $1 \%$ ciclopirox), are effective in scalp SD by reducing inflammation, scaling, and itch when compared to placebo $[14,15]$. However, their prolonged application is associated with some disadvantages, including skin atrophy, folliculitis, and tachyphylaxis for corticosteroids, and irritant contact dermatitis for antifungals such as ketoconazole [16]. Therapeutic options such as topical noncorticosteroid, antiinflammatory/antifungal combinations may represent a promising therapeutic approach as evidenced by some clinical trials $[11-13,17-$ 20].

One of the Cochrane reviews also highlighted that better outcome measures are needed to improve the evidence base for SD treatment [15]. So far, the most common therapeutic monitoring for SD is based on clinical assessment, although the identification of minimal changes may sometimes be difficult. Scalp dermoscopy or trichoscopy, which allows a magnified view of the scalp with image capture/storage facility, is a noninvasive tool for the diagnosis and treatment monitoring that has shown to be useful in some inflammatory scalp disorders [21-23].

The aim of this 1:1 sequentially randomized, controlled, investigator-single-blind clinical trial was to assess, by clinical and trichoscopic examination, the efficacy and tolerability of a combination noncorticosteroid, antiinflammatory/antifungal shampoo compared to $1 \%$ ketoconazole shampoo in the treatment of adult patients with mild-to-moderate scalp SD.

Therapeutic Monitoring of Scalp

Seborrheic Dermatitis with Trichoscopy
Table 1. Demographic characteristics and anamnestic data

\begin{tabular}{lll}
\hline & Group A & Group B \\
\hline Sex & & \\
$\quad$ Female & 4 & 5 \\
$\quad$ Male & 6 & 5 \\
Age, years & & \\
$\quad$ Mean & 40.2 & 41.5 \\
$\quad$ Range & $16-52$ & $17-54$ \\
SD clinical severity (baseline) & & \\
$\quad$ Mild & 5 & 5 \\
$\quad$ Moderate & 5 & 5 \\
\hline SD duration & 6 & 5 \\
$\quad$ 5 years & 3 & 3 \\
$\quad$ 6-10 years & 1 & 2 \\
$\quad \geq 10$ years & 7 & 6 \\
Duration of current episode, months & $5-9$ \\
$\quad$ Mean & $5-9$ & 5 \\
$\quad$ Range & & 5 \\
Pattern of disease & 4 & \\
$\quad$ Persistent & 6 & \\
$\quad$ Intermittent & & \\
\hline
\end{tabular}

\section{Materials and Methods}

Study Design and Methodology

This clinical investigation was performed in accordance with Good Clinical Practices and the Declaration of Helsinki 1996.

Twenty patients attending the Dermatology Clinic of the University of Catania from November 2013 to April 2014 and affected by mild-to-moderate scalp SD were enrolled. Inclusion criteria were age $>16$ years, 2 -week wash-out from topical antifungals/corticosteroids, and 4-week wash-out from oral antifungals/corticosteroids and hormonal therapy. Exclusion criteria were recent sun exposure and pregnancy. A written informed consent was obtained from each subject before the study procedures were conducted.

The patients were randomized into two groups ( $\mathrm{A}$ and $\mathrm{B}$ ). $\mathrm{Pa}$ tients of group A (10 cases) used a combination shampoo containing piroctone olamine, Vitis vinifera, lactic acid, lactoferrin, dipotassium glycyrrhizate, and telmesteine (Sebclair ${ }^{\circledR}$ shampoo, Sinclair Pharmaceuticals Ltd., Godalming, UK), while patients of group B (10 cases) used 1\% ketoconazole shampoo (Triatop ${ }^{\circledR} 1 \%$ shampoo, Janssen Cilag SpA). All patients applied the shampoo 3 times a week every other day for 8 weeks. A 1-month follow-up after the end of treatment was carried out for both groups of patients. To reduce potential confounding issues, all subjects were assessed by the same physician investigator (dermatologist).

\section{Subject Demographics}

Subject demographic information and anamnestic data are shown in table 1.

\section{Clinical Evaluation}

Efficacy was assessed by measuring the degree of scaling and pruritus at baseline, weeks 4 and 8 . Scaling was evaluated by $\times 20$ 
Table 2. Study results: trichoscopic and clinical evaluation (scaling, pruritus)

\begin{tabular}{|c|c|c|c|c|c|c|}
\hline & \multicolumn{2}{|l|}{ Baseline } & \multicolumn{2}{|l|}{4 weeks } & \multicolumn{2}{|l|}{8 weeks } \\
\hline & group A & group B & group A & group B & group A & group B \\
\hline \multicolumn{7}{|c|}{ Trichoscopic and clinical evaluation } \\
\hline Scaling $\left(\right.$ median $\left.^{\mathrm{a}}\right)$ & 2.5 & 2.5 & $1.5(\mathrm{p}<0.01)$ & $1.5(\mathrm{p}<0.01)$ & $0.5(\mathrm{p}<0.01)$ & $0.5(\mathrm{p}<0.01)$ \\
\hline Pruritus (median ${ }^{\mathrm{a}}$ ) & 70 & 70 & $30(\mathrm{p}<0.01)$ & 60 (n.s.) & $20(\mathrm{p}<0.01)$ & $30(\mathrm{p}<0.01)$ \\
\hline
\end{tabular}

n.s. = Not significant. ${ }^{a}$ Median values indicate numerical values separating the higher half of a data sample, a population, or a probability distribution from the lower half.

trichoscopy (Hi-Scope KH-2200, Hirox Co. Ltd., Tokyo, Japan) using a 4-point scale: $0=$ none (no desquamation); $1=$ mild (few small loose white flakes); 2 = moderate (several small to large loose white flakes), and 3 = severe (many large adherent white flakes). The measurement of pruritus was carried out by a subject-completed Visual Analogue Scale: 0 = no pruritus; $100 \mathrm{~mm}=$ severe pruritus.

Additionally, a Physician Global Assessment (PGA) was conducted at the end of the study using a 6-point scale: complete response (>90\% improvement); excellent response (70-90\% improvement); moderate response (40-69\% improvement); mild response ( $<40 \%$ improvement); no response (no change), and worsening.

An investigator evaluation of product tolerability was carried out with the help of a 4 -point scale: $0=$ very poor; $1=$ poor; $2=$ good, and 3 = excellent. In addition, subjective cosmetic acceptability was evaluated at week 4 by a 3 -point scale: $0=$ poor; $1=$ good, and 2 = excellent.

\section{Study Endpoints}

The primary endpoint was the efficacy evaluation of the tested products at weeks 4 and 8 . The secondary endpoint was the evaluation of tolerability and cosmetic acceptability.

\section{Statistical Analysis}

Data were analyzed using the Kruskal-Wallis nonparametric analysis of variance (ANOVA) test. Analyses were conducted using the SPSS statistical software package $(\mathrm{p}<0.05)$.

\section{Results}

All participants completed the study. At 4 weeks, there was a significant reduction of scaling from baseline for both groups (group A: median from 2.5 to $1.5 ; \mathrm{p}<0.01$; group B: median from 2.5 to $1.5 ; \mathrm{p}<0.01$; table 2 ). Pruritus showed a significant reduction only for group A (median from 70 to $30 ; \mathrm{p}<0.01$; table 2).

After 8 weeks, there was a significant reduction from baseline of scaling (group A: median from 2.5 to $0.5 ; \mathrm{p}<$ 0.01 ; group $B$ : median from 2.5 to $0.5 ; \mathrm{p}<0.01$; fig. 1,2 ) and of pruritus (group A: median from 70 to 20; $<<0.01$;
Table 3. Study results: PGA and tolerability

\begin{tabular}{lll}
\hline & \multicolumn{2}{c}{8 weeks } \\
\cline { 2 - 3 } & group A & group B \\
\hline PGA & & \\
Complete response & $90 \%$ & $90 \%$ \\
Excellent response & $10 \%$ & $10 \%$ \\
\hline Tolerability & & \\
Excellent & $95 \%$ & $80 \%$ \\
Good & $5 \%$ & $20 \%$ \\
\hline
\end{tabular}

group B: median from 70 to $30 ; \mathrm{p}<0.01$ ) for both groups (table 2).

PGA showed a complete response in $90 \%$ of the cases for both groups. Overall, tolerability was rated as excellent (no sign of irritation) in $95 \%$ of the patients in group A and in $80 \%$ of the patients in group B (table 3). The cosmetic acceptability was rated as excellent in $90 \%$ of the subjects in group A and $70 \%$ of the subjects in group B (table 3 ).

The efficacy seen at the end of treatment was maintained for both groups at the 1-month follow-up.

\section{Discussion}

Scalp conditions with visible flaking, such as SD, have a negative impact on the patient's quality of life and should be properly treated [8]. In our study, the noncorticosteroid combination shampoo has shown to be a safe and effective treatment approach for $90 \%$ of the patients with mild-to-moderate SD and, in particular, was noninferior to $1 \%$ ketoconazole shampoo. Ketoconazole shampoo in concentrations ranging from 1 to $2 \%$ is considered one of the most effective treatments for scalp SD as confirmed by several randomized controlled trials [15]. 
Fig. 1. Trichoscopy evaluation of a noncorticosteroid combination shampoo in the treatment of mild scalp SD at baseline (a) and after 8 weeks (b): complete response.

Fig. 2. Trichoscopy evaluation of a noncorticosteroid combination shampoo in the treatment of moderate scalp SD at baseline (a) and after 8 weeks (b): complete response.
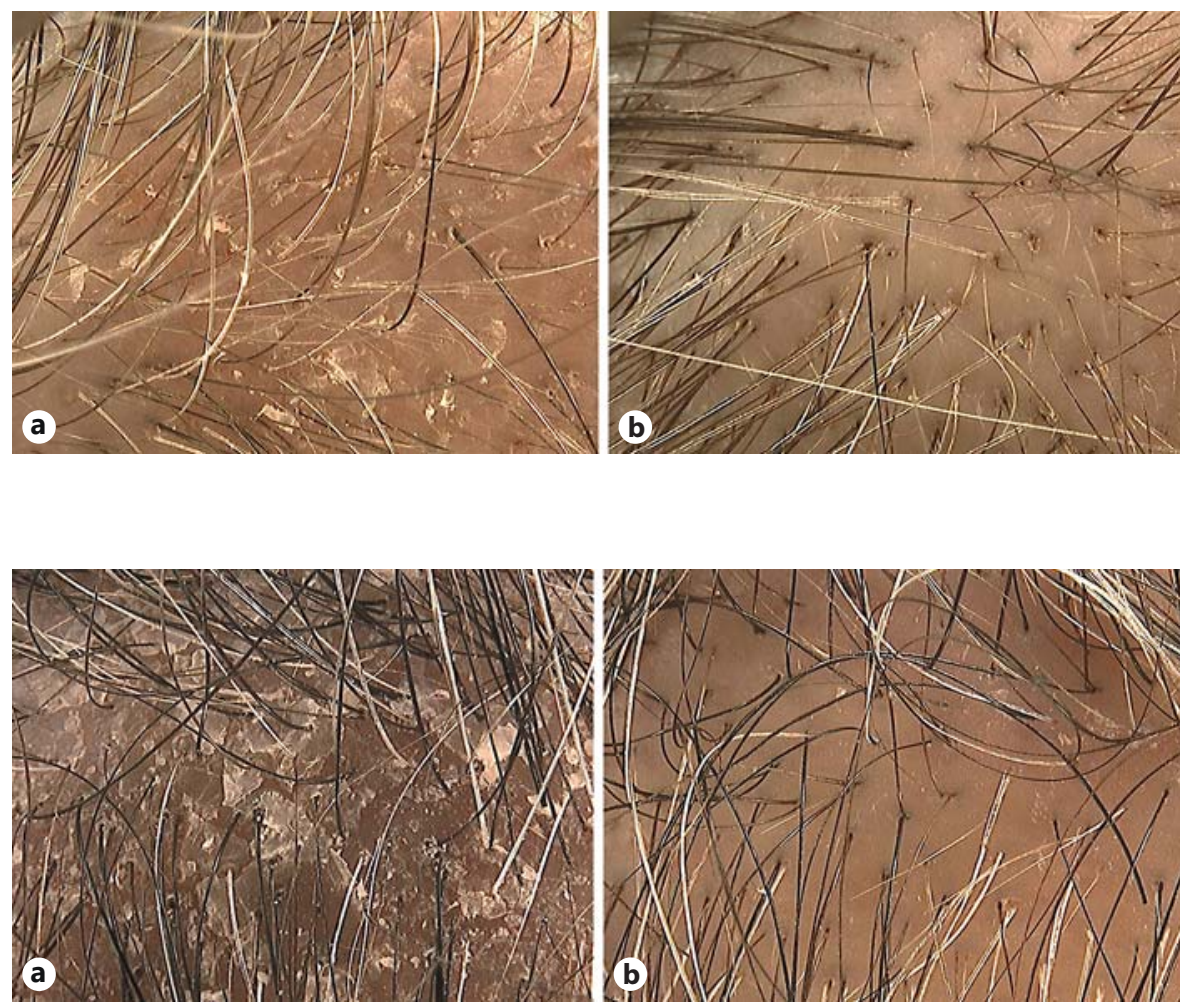

Although both tested products proved to be safe and efficacious for SD after 8 weeks, the combination shampoo was assessed as better than ketoconazole to control pruritus at 4 weeks. Moreover, the efficacy for both groups was maintained at the 1-month follow-up after therapy discontinuation.

The effect of the combination shampoo in SD may be related to multiple mechanisms of action of the active ingredients. Piroctone olamine is a well-known antifungal agent that may act on Malassezia spp. by chelation of iron and other elements. V. vinifera (grapevine) is able to block endothelial oxidative damage, and lactic acid is an effective keratolytic enhancing the desquamation of upper layer corneocytes. Finally, lactoferrin is a glycoprotein with recognized antimicrobial activity (bactericide and fungicide), dipotassium glycyrrhizate (extracted from liquorice root) has antiinflammatory, antioxidative, and antiirritant properties, and telmesteine exhibits antiinflammatory, soothing, and antipruritic effects $[12,24]$. The product is fragrance free, and its vehicle is formulated with some ingredients, such as olive oil and glycerin, for an additional moisturizing effect.

In our study, treatment outcome has been evaluated by trichoscopy, a technique that has demonstrated to allow

Therapeutic Monitoring of Scalp

Seborrheic Dermatitis with Trichoscopy for a simple, more accurate evaluation of scaling severity in patients with scalp psoriasis [25]. So far, no published studies using trichoscopic evaluation of SD treatment outcome are available.

In conclusion, the results of our study demonstrate that the combination noncorticosteroid, antiinflammatory/antifungal shampoo represents an alternative to standard topical treatment in the management of scalp SD. Moreover, trichoscopy proves to be an accurate and reliable therapeutic monitoring method for acquisition of quantifiable data during treatments of scalp SD. Further studies on a higher number of patients and with a longterm follow-up are needed in order to consolidate the approach proposed by our research.

\section{Statement of Ethics}

This clinical investigation was performed in accordance with Good Clinical Practices and the Declaration of Helsinki 1996.

\section{Disclosure Statement}

The authors declare no conflicts of interest. 


\section{References}

1 Gupta AK, Bluhm R: Seborrheic dermatitis. J Eur Acad Dermatol Venereol 2004;18:13-26.

2 Stefanaki I, Katsambas A: Therapeutic update on seborrheic dermatitis. Skin Therapy Lett 2010;15:1-4.

3 Del Rosso JQ: Adult seborrheic dermatitis: a status report on practical topical management. J Clin Aesthet Dermatol 2011;4:32-38.

4 Hay RJ: Malassezia, dandruff and seborrhoeic dermatitis: an overview. Br J Dermatol 2011; 165:2-8.

5 Bukvić Mokos Z, Kralj M, Basta-Juzbašić A, Lakoš Jukić I: Seborrheic dermatitis: an update. Acta Dermatovenerol Croat 2012;20 98-104.

6 Dessinioti C, Katsambas A: Seborrheic dermatitis: etiology, risk factors, and treatments: facts and controversies. Clin Dermatol 2013; 31:343-351.

7 Clark GW, Pope SM, Jaboori KA: Diagnosis and treatment of seborrheic dermatitis. Am Fam Physician 2015;91:185-190.

8 Grimalt R: A practical guide to scalp disorders. J Investig Dermatol Symp Proc 2007; 12: 10-14.

9 Hald M, Arendrup MC, Svejgaard EL, Lindskov R, Foged EK, Saunte DM: Evidence-based Danish guidelines for the treatment of Malassezia-related skin diseases. Acta Derm Venereol 2015;95:12-19.

10 Gary G: Optimizing treatment approaches in seborrheic dermatitis. J Clin Aesthet Dermatol 2013;6:44-49.

11 Naldi L: Seborrhoeic dermatitis. BMJ Clin Evid 2010;2010:1713.
12 Bhatia N: Treating seborrheic dermatitis: review of mechanisms and therapeutic options. J Drugs Dermatol 2013;12:796-798.

13 David E, Tanuos H, Sullivan T, Yan A, Kircik LH: A double-blind, placebo-controlled pilot study to estimate the efficacy and tolerability of a nonsteroidal cream for the treatment of cradle cap (seborrheic dermatitis). J Drugs Dermatol 2013;12:448-452.

14 Kastarinen H, Oksanen T, Okokon EO, Kiviniemi VV, Airola K, Jyrkkä J, Oravilahti T, Rannanheimo PK, Verbeek JH: Topical antiinflammatory agents for seborrhoeic dermatitis of the face or scalp. Cochrane Database Syst Rev 2014;5:CD009446.

15 Okokon EO, Verbeek JH, Ruotsalainen JH, Ojo OA, Bakhoya VN: Topical antifungals for seborrhoeic dermatitis. Cochrane Database Syst Rev 2015;5:CD008138.

16 Liu J, Warshaw EM: Allergic contact dermatitis from ketoconazole. Cutis 2014;94:112114.

17 Veraldi S, Menter A, Innocenti M: Treatment of mild to moderate seborrhoeic dermatitis with MAS064D (Sebclair), a novel topical medical device: results of a pilot, randomized, double-blind, controlled trial. J Eur Acad Dermatol Venereol 2008;22:290-296.

18 Nalamothu V, O’Leary AL, Kandavilli S, Fraser J, Pandya V: Evaluation of a nonsteroidal topical cream in a guinea pig model of Malassezia furfur infection. Clin Dermatol 2009; 27:S41-S43.

19 Kircik L: An open-label, single-center pilot study to determine the antifungal activity of a new nonsteroidal cream (Promiseb Topical Cream) after 7 days of use in healthy volunteers. Clin Dermatol 2009;27:S44-S47.
20 Elewski B: An investigator-blind, randomized, 4-week, parallel-group, multicenter pilot study to compare the safety and efficacy of a nonsteroidal cream (Promiseb Topical Cream) and desonide cream $0.05 \%$ in the twice-daily treatment of mild to moderate seborrheic dermatitis of the face. Clin Dermatol 2009;27:S48-S53.

21 Lacarrubba F, Dall'Oglio F, Nasca MR, Micali G: Videodermatoscopy enhances diagnostic capability in some forms of hair loss. Am J Clin Dermatol 2004;5:205-208.

22 Nikam VV, Mehta HH: A nonrandomized study of trichoscopy patterns using nonpolarized (contact) and polarized (noncontact) dermatoscopy in hair and shaft disorders. Int J Trichology 2014;6:54-62.

23 Kibar M, Aktan Ş, Bilgin M: Dermoscopic findings in scalp psoriasis and seborrheic dermatitis; two new signs; signet ring vessel and hidden hair. Indian J Dermatol 2015;60:4145.

24 Veraldi S, De Micheli P, Schianchi R, Lunardon L: Treatment of pruritus in mild-to-moderate atopic dermatitis with a topical non-steroidal agent. J Drugs Dermatol 2009;8:537539 .

25 Rossi A, Pranteda G, Iorio A, Mari E, Milani M: Efficacy of Iralfaris shampoo in the treatment of scalp psoriasis: a videodermoscopy evaluation prospective study in 70 patients. G Ital Dermatol Venereol 2012;147:625-630. 